\title{
Narrativas autoetnográficas e desafios para a educação física nos Estudos Culturais Físicos
}

\author{
Ariane Boaventura da Silva Sá' (D) \\ Vitor Hugo Maranil (D) \\ Larissa Michelle Lara' (D)
}

\section{RESUMO}

Este estudo explora os Estudos Culturais Físicos como um campo de interlocução para o desenvolvimento de uma investigação colaborativa e teoricamente informada, que possa contribuir para o ensino e a pesquisa na educação física brasileira. A partir da abordagem autoetnográfica, revisitamos as maneiras pelas quais experiências distintas com os Estudos Culturais Físicos tocaram (e tocam) nossas identidades, imprimindo marcas que nos (re)constroem em ações de docência, pesquisa e intervenção social. As narrativas que decorrem desse esforço colaborativo carregam desafios que (re)significam experiências ou aspectos anteriormente inexplorados em nossas subjetividades, oferecendo-nos a possibilidade de repensar e reestruturar nossas práticas de embodiment. Por fim, ao compartilharmos nossas narrativas na interlocução com os Estudos Culturais Físicos, entendemos esse campo (epistemológico, ontológico e metodológico) como profícuo para o diálogo com a educação física no contexto brasileiro.

PALAVRAS-CHAVE

Estudos Culturais Físicos; cultura; embodiment; autoetnografia. 


\title{
AUTOETHNOGRAPHIC NARRATIVES AND CHALLENGES FOR PHYSICAL EDUCATION IN PHYSICAL CULTURAL STUDIES
}

\begin{abstract}
This study explores Physical Cultural Studies as a field of dialogue for developing collaborative and theoretically informed research that can contribute to teaching and research experiences in Brazilian physical education. From the autoethnographic approach, the ways in which different experiences with the Physical Cultural Studies touched our identities were revisited, imprinting marks that (re)build us in our teaching, research, and intervention in social reality. The narratives that result from this joint and collaborative effort carry challenges that (re)signify experiences or aspects previously unexplored in our subjectivities, offering us the possibility to rethink and restructure our embodiment. Finally, besides sharing our narratives in the dialogue with Physical Cultural Studies, this field (epistemological, ontological, and methodological) is useful for dialogue with physical education in the Brazilian context.
\end{abstract}

KEYWORDS

Physical Cultural Studies; culture; embodiment; autoethnography.

\section{NARRATIVAS AUTETNOGRÁFICAS Y DESAFIOOS PARA LA EDUCACIÓN FÍSICA EN ESTUDIOS FISICO CULTURALES}

\section{RESUMEN}

Este estudio explora los Estudios Culturales Físicos como un campo de diálogo para el desarrollo de investigaciones colaborativas y teóricamente informadas, que pueden contribuir a la enseñanza y a la investigación en educación física brasileña. Desde el enfoque autoetnográfico, revisamos las formas en que diferentes experiencias con que Estudios Culturales Físicos han tocado nuestras identidades, imprimiendo marcas que nos reconstruyen en acciones de enseñanza, investigación e intervención social. Las narrativas que resultan de este esfuerzo colaborativo conllevan desafíos que resignifican experiencias o aspectos previamente inexplorados en nuestras subjetividades, ofreciéndonos la posibilidad de repensar y reestructurar nuestro embodiment. Finalmente, mientras compartimos nuestras narrativas en el diálogo con Estudios Culturales Físicos, entendemos que este campo (epistemológico, ontológico y metodológico) es útil para el diálogo con la educación física en el contexto brasileño.

Estudios Culturales Físicos; cultura; embodiment; autoetnografía. 


\section{INTRODUÇÃO}

Estudos Culturais Físicos ${ }^{1}$ - tradução de physical cultural studies (PCS) é a terminologia usada para um campo de estudos em processo de construção e consolidação (Andrews, 2008; Silk e Andrews, 2011; Andrews e Silk, 2015; Silk, Andrews e Thorpe, 2017) que tem orientado o trabalho de pesquisadores comumente vinculados a universidades nos Estados Unidos, no Reino Unido, no Canadá, na Austrália e na Nova Zelândia. De acordo com Lara e Rich (2017, p. 1312), PCS é um termo "polêmico e nebuloso que tem sido referenciado diversamente pela respectiva literatura como projeto, formação intelectual, quadro, movimento, campo de investigação, sensibilidade, mandato e abordagem". Essa diversidade de interpretações do que seria o PCS pode ser lida como salutar à medida que esse projeto se encontra em desenvolvimento a partir de discussões que envolvem o corpo (in)ativo e a cultura física, em meio a diferentes contextos e relações de poder.

É difícil afirmar quando e onde, exatamente, surgem os Estudos Culturais Físicos, pois não existe um consenso entre os pesquisadores (Silk, Andrews e Thorpe, 2017). Entretanto, em linhas gerais, é possível dizer, com base em Lara et al. (2019), que esse campo de estudos se desenvolveu tanto como desdobramento dos Estudos Culturais surgidos na Inglaterra, no período pós-guerra, quanto por consequência das lutas disciplinares nos departamentos de cinesiologia, ocorridas nas últimas décadas, nos Estados Unidos. As bases do PCS, "lançadas há mais de 20 anos, não contêm histórias ou trajetórias disciplinares fixas; pelo contrário, elas apresentam tradições empíricas, teóricas ou metodológicas diversas" (Lara et al., 2019, p. 229).

Silk, Andrews e Thorpe (2017) esclarecem que o PCS se constitui como projeto político em que os pesquisadores visam compreender os efeitos do poder e das relações de poder, uma vez que partem do pressuposto de que as sociedades são organizadas por linhas de diferenciação hierárquicas, tais como: classe, etnia, gênero, capacidade, geração, nacionalidade, raças e/ou sexualidade. Essas diferenças de hierarquia se manifestam na existência de desigualdades ou injustiças culturais, vantagens ou desvantagens, habilitações ou restrições, empoderamentos ou desempoderamentos. Por isso, os/as pesquisadores/as do PCS, comprometidos/as com a progressiva mudança social, engajam-se criticamente a partir da cultura física, considerando-a como o local onde tais divisões são encenadas, experimentadas e, às vezes, contestadas.

$\mathrm{Na}$ tentativa de contribuir para o entendimento da abordagem do PCS, Andrews (2008, p. 54-55, tradução nossa) assim descreve:

Os Estudos Culturais Físicos avançam na análise crítica e teórica da cultura física, em todas as suas inumeráveis formas. Estas incluem práticas relacionadas ao esporte, ao exercício, à saúde, à dança e ao movimento, que a pesquisa do PCS

1 A tradução de Physical Cultural Studies (PCS) para estudos culturais físicos ocorre como possibilidade de aproximação e diálogo com esse campo a partir de nossos processos identitários no contexto brasileiro. Apesar de realizarmos a tradução do termo, manteremos, no texto, a sigla PCS, como costumeiramente esse campo é referendado por pesquisadores/as que têm incursionado por ele. 
localiza e analisa dentro dos contextos social, político, econômico e tecnológico mais amplos, nos quais estão inseridos. Especificamente, o PCS dedica-se à compreensão baseada no contexto das práticas, dos discursos e das subjetividades corporais, através dos quais os corpos ativos se tornam organizados, representados e experimentados, em relação às operações de poder social. O PCS identifica, assim, o papel desempenhado pela cultura física na reprodução e, às vezes, desafiadora, de normas e diferenças específicas de classe, etnia, de gênero, de capacidade, de gerações, de nacionalidades, de raças e/ou de sexualidades. Por meio do desenvolvimento e disseminação estratégica de formas potencialmente empoderadoras de conhecimento e compreensão, o PCS procura iluminar e intervir em locais de injustiça e desigualdade cultural. Além disso, como a cultura física é tanto manifestada, quanto vivenciada em diferentes formas, o PCS adota uma abordagem multimétodo para engajar o empírico (incluindo etnografia e autoetnografia, observação participante, análise de discurso e mídia e análise contextual). O PCS avança num vocabulário teórico igualmente fluido, utilizando conceitos e teorias de uma variedade de disciplinas (incluindo estudos culturais, economia, história, estudos de mídia, filosofia, sociologia e estudos urbanos) para envolver e interpretar o aspecto particular da cultura física sob escrutínio.

Os Estudos Culturais Físicos incorporam uma compreensão relacional e pluralista acerca da cultura física, cujas expressões do active embodiment ${ }^{2}$ são experimentadas e examinadas a partir de processos socioestruturais, discursivos, institucionais, comunitários, subjetivos e corpóreos (Silk, Andrews e Thorpe, 2017). Nessa perspectiva, esse projeto avança em investigações críticas e empiricamente fundamentadas, teoricamente informadas, politicamente incisivas e metodologicamente rigorosas acerca da cultura física, o que inclui práticas esportivas, exercícios de condicionamento físico, saúde, recreação, danças, entre outras, localizadas e analisadas em contextos político, econômico e tecnológico nos quais estão situadas socialmente (Andrews, 2008; Silk e Andrews, 2011).

Diante da complexidade desse campo investigativo, os Estudos Culturais Físicos, como têm sido traduzidos para a língua portuguesa (Lara e Rich, 2017; Lara, 2019; Marani, 2019; Marques, 2019; Sá, 2019), tornaram-se o foco de estudos sistemáticos realizados pelo Grupo de Pesquisa Corpo, Cultura e Ludicidade (GPCCL), na Universidade Estadual de Maringá (UEM). A aproximação com tais leituras despertou interesses que nos levaram a vivenciar experiências práticas, tais como a utilização da abordagem do PCS em ações de docência e, até mesmo, o contato direto com pesquisadores/as que integram esse campo.

2 Embodiment é um termo complexo, sem a existência de uma palavra específica na língua portuguesa que consiga dimensioná-lo. Em linhas gerais, pode ser compreendido como um corpo vivido, experimentado, incorporado (Lara e Rich, 2017); um corpo consciente, que se percebe holisticamente. Um movimento embodied pode ser entendido "como uma prática social que está profundamente entrelaçada com o contexto histórico, sociocultural, político e relações de poder econômico que moldam a vida cotidiana" (Fullagar, 2019, p. 65, tradução nossa). 
A partir de leituras e análises de produções teóricas orientadas pelos Estudos Culturais Físicos é que desenvolvemos este estudo. Exploramos o PCS como um campo de interlocução para uma investigação colaborativa e teoricamente informada. Para isso, optamos por utilizar uma abordagem com narrativas autoetnográficas ${ }^{3}$, em que revisitamos as maneiras pelas quais experiências distintas com o PCS tocaram (e tocam) nossas identidades, imprimindo marcas que nos (re)constroem em nossas ações de docência, de pesquisa e de intervenção na realidade social.

A pesquisa autoetnográfica procura situar o "eu" como protagonista da narrativa de pensamentos e interpretações relacionados a determinados acontecimentos (Markula e Silk, 2011). E a partir desse relato narrativo que vozes dos/as pesquisadores/as são celebradas como maneiras de apresentar experiências que se tornam organizadas, a partir de uma totalidade significativa, destacando elementos biográficos e relações sociais que integram os acontecimentos narrados (Markula e Denison, 2005; Rego, 2014; Calha, 2017; Silva, Oliveira e Souza, 2018). Tem-se, nessa perspectiva, a ênfase no papel ativo do/a pesquisador/a, afastando-o/a de uma escrita neutra realizada em terceira pessoa que, comumente, insere-o/a como observador/a objetivo/a e distante das experiências as quais se preocupa relatar (Markula e Denison, 2005).

Apoiados na autoetnografia, procuramos construir reflexões que oportunizem ao/à leitor/a o acesso às formas pelas quais nossas posições acadêmicas foram deflagradas, em meio às experiências que contribuíram para nossos relatos. Nesse sentido, a ideia é que sejam realizados insigths da nossa trajetória em diversas experiências com o PCS, organizadas a partir de três tópicos que visam aprofundar tais discussões sob diferentes perspectivas, quais sejam: incursões iniciais pelo PCS, por meio do estágio pós-doutoral na Universidade de Bath, no Reino Unido; experiências pedagógicas construídas a partir da sensibilidade do PCS em uma disciplina de graduação ofertada localmente em uma universidade federal brasileira; e, por fim, elementos de uma entrevista realizada com o Prof. Dr. David L. Andrews, durante visita institucional realizada na Universidade de Maryland, nos Estados Unidos.

As narrativas que decorrem desse esforço conjunto e colaborativo nos possibilitam repensar e reestruturar nossas práticas de embodiment e nossos modos de fazer pesquisa. Ao longo de cada tópico, são ofertados elementos que nos auxiliam na constituição dos relatos autobiográficos, a partir das experiências vivenciadas. $\mathrm{Na}$ sequência, realizamos algumas reflexões que nos auxiliam a pensar em maneiras pelas quais os Estudos Culturais Físicos podem contribuir para qualificar debates na educação física, no contexto brasileiro.

3 Pesquisas autoetnográficas têm ocupado papel relevante nos estudos culturais físicos, pois possibilitam relações constantes entre texto e corpo, escrita e experiência, marcados por elementos autorreflexivos e políticos que atravessam a autoria acadêmica. Como exemplos de investigações autoetnográficas no PCS, citamos: Giardina e Newman (2011a); Thorpe, Barbour e Bruce (2011); King-White (2012); Smith (2017); Thorpe e Marfell (2019). 


\section{INCURSÕES INICIAIS PELOS ESTUDOS CULTURAIS FÍSICOS PARA A CONSTRUÇÃO DE UM DIÁLOGO PROFÍCUO}

Larissa Lara - O interesse em pesquisar temáticas voltada ao corpo e à cultura na educação física a partir da interlocução com as ciências humanas e sociais é resultado de 22 anos de pesquisa, sendo 15 deles dedicados à coordenação do Grupo de Pesquisa Corpo, Cultura e Ludicidade, junto à Universidade Estadual de Maringá, vinculado ao Conselho Nacional de Ensino e Pesquisa (CNPq). Nesse percurso, o corpo, em sua gestualidade e práticas de embodiment, foi investigado na religiosidade afro-brasileira, na cultura popular, em comunidades quilombolas e na produção de conhecimento na educação física latino-americana, com aporte de referenciais da Teoria Crítica, da Antropologia Social e da Sociologia (notadamente, dos Estudos Culturais britânicos e latino-americanos). Recentemente, tenho orientado minhas investigações por referenciais ligados aos Estudos Culturais Físicos, motivada pelos estudos desenvolvidos no estágio sênior pós-doutoral ${ }^{4}$, na Universidade de Bath, Reino Unido (outubro de 2016 a julho de 2017), com a colaboração da professora Emma Rich 5 .

Ao tomar conhecimento do Grupo de Pesquisa Physical Cultural Studies ${ }^{6}$ (PCS/ Bath), na Universidade de Bath, visualizei-me em seu compromisso de identificar e intervir nas distintas culturas físicas em relação a gênero, deficiência, etnia, raça, classe social, sexualidade, examinando as relações entre atividade física, saúde, esporte e bem-estar com a cultura, a tecnologia, o ambiente, o espaço urbano, as pedagogias, a pobreza, as desigualdades e as relações de poder. Inquietou-me, de modo bastante positivo, o modo como o conceito de cultura física apareceu delineado no referido campo de investigação, estruturado a partir de teorias basilares ao grupo, entre as quais, os Estudos Culturais, que também têm orientado pesquisas que desenvolvo no Brasil. Nessas incursões, chamou-me a atenção a estruturação do conceito de cultura física junto aos Estudos Culturais Físicos, ligado não a uma cultura da fisicalidade, mas entendido e desenvolvido densamente em sua dimensão social, discursiva, corpórea, bem como em suas relações de poder, dominação e resistência ${ }^{7}$.

O meu contato inicial com o PCS deu-se com o texto de Giardina e Newman (2011b), intitulado "What is this 'physical' in Physical Cultural Studies?". Os autores iniciam suas reflexões com a menção às contribuições de Ingham (1997) e Andrews (2008) no tocante ao PCS, referindo-se ao chamado para estudos críticos na cultura física.

4 O estágio pós-doutoral desenvolvido na Universidade de Bath contou com o apoio da Coordenação de Aperfeiçoamento de Pessoal de Nível Superior (CAPES-Programas Estratégicos) - Código de Financiamento 001.

5 Emma Rich é professora no Department for Health, Universidade de Bath/UK.

6 O Grupo de Pesquisa Physical Cultural Studies, criado em 2008, na Universidade de Bath, recentemente alterou o seu nome para Physical Culture, Sport and Health, de modo a responder a novos anseios decorrentes do grupo de pesquisadores que o integram. Sobre o Grupo, ver: http://www.bath.ac.uk/health/research/physical-culture-sport-health/. Acesso em: 16 jul. 2019.

7 Em complemento, considero importante mencionar o fato de o PCS vincular-se ao Departamento de Saúde, ligado à Facultie Humanities \& Social Sciences, na Universidade de Bath, algo que remete ao entendimento de saúde numa perspectiva ampliada. 
Essa leitura introdutória ao texto foi a motivação para investigar como era construída e desenvolvida uma episteme do corpo e das práticas corporais a partir do PCS, o que culminou na organização do plano de trabalho para o desenvolvimento do estágio pós-doutoral, no Reino Unido. Uma segunda aproximação com esse campo deu-se pelo acesso digital à palestra desenvolvida na Universidade de São Paulo (USP) por David Andrews, em que o pesquisador apresentou reflexões constantes da publicação "Kinesiology's inconvenient truth: the physical cultural studies imperative" (Andrews, 2008), um dos principais textos que impulsionam o chamado para a organização de um conjunto de pesquisadores da sociologia do esporte engajados em disseminar e consolidar o PCS como um projeto intelectual e de intervenção na realidade social.

O plano de trabalho proposto para o estágio pós-doutoral considerava essas interlocuções iniciais, ao mesmo tempo em que propunha enfrentar os desafios advindos da colaboração acadêmica no exterior. $\mathrm{O}$ ensejo investigativo era tanto conhecer o projeto Estudos Culturais Físicos (em sua origem e contribuições epistêmicas, ontológicas e metodológicas) quanto o trabalho desenvolvido pelo grupo PCS/Bath. Parti do pressuposto de que os achados da pesquisa junto ao $\mathrm{PCS}$, no confronto com a realidade da educação física brasileira, poderiam contribuir para desdobramentos em seu campo epistemológico, sobretudo na chamada subárea sociocultural e pedagógica. Mas o encontro com o PCS foi muito mais intenso do que eu mesma supunha. Identifiquei-me diretamente com formas de investigação que dialogavam com os estudos culturais, assim como com a teoria queer, os estudos de mídia, a sociologia do corpo, a sociologia do esporte, entre outros, por meio de discursos, práticas de embodiment e intervenção na realidade social pautados pelo acesso democrático e justo à cultura física.

A interlocução com o PCS, iniciada em 2016, teve continuidade a partir de algumas ações institucionais. Uma delas diz respeito ao trato com esse conhecimento na pós-graduação (mestrado e doutorado), junto ao Programa de Pós-graduação Associado em Educação Física da Universidade Estadual de Maringá (UEM) e da Universidade Estadual de Londrina (UEL), por meio da oferta da disciplina Tópicos Especiais - Physical Cultural Studies: leituras contemporâneas em corpo, saúde e esporte, em 2018 e 2019. Outra ação diz respeito ao estudo sistemático de publicações relacionadas ao $\mathrm{PCS}$ em nosso grupo de pesquisa (GPCCL/UEM/CNPq), com a participação de pesquisadores/as, pós-graduandos/as, alunos/as de iniciação à pesquisa e estudantes de graduação. $\mathrm{O}$ estudo, realizado nesse grupo, motivou alguns de meus/minhas orientandos/as de doutorado a desenvolverem suas teses com aporte dos Estudos Culturais Físicos e a buscarem interlocuções com pesquisadores/as afiliados/as a esse campo investigativo, em universidades no exterior, processo que apenas se inicia junto à Universidade de Maryland, com a colaboração do professor David Andrews ${ }^{8}$.

A interlocução com a produção teórica ligada ao PCS também intensificou a minha prática de embodiment como professora e pesquisadora em uma instituição pública de ensino superior no Brasil. A defesa de uma pós-graduação que não seja focada no produtivismo acadêmico, mas em uma produção de qualidade, com tempo

8 David Andrews é professor/pesquisador no Department of Kinesiology, University of Maryland/USA. 
para ser desenvolvida e com capacidade de intervir na realidade social, passou a ser fortalecida com as contribuições de Silk, Francombe-Webb e Andrews (2014), ao proporem um "slowing social science", e com o texto de Silk, Bush e Andrews (2010), ao realizem uma crítica social à universidade e à ciência na perspectiva de mercado, situando a crise da sociologia do esporte nesse contexto.

Atualmente, em virtude dos desastrosos discursos, práticas e políticas governamentais brasileiras (federais e estaduais) que violam direitos constitucionais dos sujeitos em relação à educação, à cultura física, à diversidade e à liberdade de expressão, a interlocução com o PCS, considerada em suas várias dimensões (Silk, Andrews e Thorpe, 2017), representa inspiração e motivação para ações voltadas ao engajamento coletivo, com vistas à defesa de uma universidade não subordinada ao pensamento privatista e a suas lógicas de mercado. Consequentemente, entendo que os Estudos Culturais Físicos, ao investigarem diferentes realidades assoladas pelo neoliberalismo e pela crescente privatização da educação, podem contribuir para despertar consciências acerca da necessidade de ações de intervenção na realidade social e que possam levar à garantia dos direitos à educação, à cultura física e à liberdade de expressão, ora ameaçados como bens públicos.

\section{INTERVENÇÕES PEDAGÓGICAS A PARTIR DOS ESTUDOS CULTURAIS FÍSICOS EM UMA UNIVERSIDADE BRASILEIRA}

Vitor Hugo Marani - Minhas experiências com os Estudos Culturais Físicos ocorreram, inicialmente, a partir do meu ingresso no curso de doutorado, em 2017, por meio do diálogo com a professora orientadora recém-chegada de seu estágio pós-doutoral na Universidade de Bath. Naquele ano, passei a incursionar por referenciais do PCS e, com isso, a ampliar meu olhar sobre esse campo de estudos? A leitura da obra Routledge Handbook of Physical Cultural Studies (Silk, Andrews e Thorpe, 2017), acrescida dos conhecimentos adquiridos em disciplina específica sobre PCS, ofertada no Programa de Pós-Graduação Associado em Educação Física UEM-UEL, oportunizaram-me o acesso a diversos escritos desse campo de estudos (Andrews, 2008; Silk e Andrews, 2011; Silk, Francombe-Webb e Andrews, 2014) e à leitura de textos disseminados, no Brasil, sobre a temática (Lara e Rich, 2017; Fullagar, 2019; Lara et al., 2019).

Do cenário apresentado, visualizei possibilidades investigativas que pudessem subsidiar minhas intervenções pedagógicas como professor no curso de educação física, da Universidade Federal de Mato Grosso - Campus Universitário do Araguaia (UFMT/CUA), no interior do Centro-Oeste brasileiro. Das leituras realizadas, os recortes que me moviam estavam direcionados às relações entre Estudos Culturais Físicos e pedagogia crítica, bem como às inovações meto-

9 Atualmente, desenvolvo o doutorado sanduíche (Bolsista PDSE/Capes - Edital no 41/2018) junto à Universidade de Maryland, sob a supervisão do Prof. Dr. David Andrews, bem como atuo como membro do Physical Cultural Studies Laboratory/School of Public Health/University of Maryland. 
dológicas ligadas às estratégias de intervenção criadas a partir de esforços de pesquisadores/as do PCS. Ao mesmo tempo, esse grupo demarcava suas críticas à carência de estudos que transcendessem os espaços das salas de aula/laboratórios em busca de uma pedagogia pública e, de fato, incorporada (King-White, 2012; Francombe-Webb, Silk e Bush, 2017).

Com as contribuições dos autores/as do PCS, pude refletir acerca da formação universitária, baseada no engajamento ativo direcionado ao diálogo democrático na sala de aula, para o desenvolvimento de uma compreensão crítica - teórica e empírica - da cultura física. Ancorado em tais pressupostos, tive a oportunidade de construir uma proposta na disciplina Tópicos em Cultura e Diversidade Étnico-racial, no ano de 2018. Voltado a experiências investigativas, o escopo apontou como diferentes dispositivos de poder operavam na sociedade contemporânea, em especial a partir de demarcadores de raça e etnia. Desse modo, a intenção foi construir conhecimentos pautados no envolvimento público dos estudantes, contribuindo para a estruturação de comunidade dialógica, baseada em um engajamento reflexivo para a mudança social (Freire, 2018a).

O curso, composto por 64 horas distribuídas em encontros semanais, foi desenhado, em um primeiro momento, a partir do estudo de categorias como cultura e identidade (Hall, 1997, 2000, 2003), educação (Giroux, 1997, 2013; Freire, 2018a, 2018b) e relações étnico-raciais a partir de documentos que norteiam tais dimensões na educação brasileira (Brasil, 1996, 2003, 2004, 2008). Posteriormente, os estudantes, em pares, foram desafiados a elaborar projetos de pesquisa a partir do modo pelo qual suas subjetividades foram tocadas ao longo das discussões realizadas nas aulas. Esses projetos deveriam envolver, especificamente, questões étnico-raciais que, de algum modo, pudessem ser incorporadas em suas agendas.

Aprofundar discussões étnico-raciais era algo necessário àquela realidade, dadas as características socioculturais da região, marcada pela presença de povos indígenas que, cotidianamente, encontram-se em condição de marginalização social. Essa exclusão chamara a atenção, especialmente, de duas estudantes (mulheres negras) que percebiam a ausência de espaços de respeito e valorização das diferenças na educação física escolar. Dessa constatação, elas procuraram compreender como esses corpos "étnico-raciais" experimentavam a educação física por meio de disputas culturais ali travadas.

Ao realizarem entrevistas com o intuito de entender como as relações de poder operavam naquele contexto, constataram que o professor de educação física não (re)conhecia as características culturais indígenas e, por isso, tinha uma representação genérica da identidade étnica pertencente a esses estudantes, o que oprimia suas práticas, inclusive corporais. Desse modo, as estudantes procuraram construir meios para desafiar as estruturas de poder social dissipadas nas aulas de educação física. A primeira etapa, baseada no diálogo, foi construída com o professor por meio do reconhecimento das narrativas étnico-raciais no contexto regional e da reflexão crítica sobre como tais narrativas poderiam servir como aporte para subverter representações de poder operantes nas aulas. Como resultado, a segunda etapa foi realizada a partir da construção de quatro aulas, elaboradas com base em uma pedagogia incorporada (embodiment), na qual os elementos identitários indí- 
genas fossem reconhecidos na educação física. A partir da experimentação de jogos, esportes, danças e lutas da etnia Xavante foi possível romper, de maneira corpórea, com identidades culturais fixas dissipadas em diferentes espaços sociais que, muitas vezes, estruturam-se, como explica Giroux (2013), de modo a marginalizar outras formas culturais.

Baseada nos pressupostos dos Estudos Culturais Físicos, da pedagogia crítica e do embodiment, a ação pedagógica realizada deu-se a partir da conscientização reflexiva e da criação de conhecimentos pautados em uma intervenção operante do envolvimento público dos estudantes com a realidade social. Dessa forma, houve conscientização acerca de relações de poder e privilégios, articulando tais apropriações a contextos sociais e culturais que se colocavam para além dos muros da universidade - a escola -, o que ofereceu espaços de interações sociais e análises em que o corpo fosse o vetor de compreensão de discussões mais amplas, especificamente a partir das relações entre educação, raça e etnia.

\section{OS ESTUDOS CULTURAIS FÍSICOS A PARTIR DA VISÃO DE DAVID ANDREWS: UMA PRIMEIRA APROXIMAÇÃO PARA A COMPREENSÃO DO CAMPO}

Ariane Boaventura da Silva Sá - Os Estudos Culturais Físicos passaram a compor meus interesses investigativos com os estudos doutorais. O primeiro contato imediato com as discussões ocorreu por meio da disciplina intitulada Physical Cultural Studies: questões contemporâneas em saúde, corpo e esporte ${ }^{10}$, realizada em julho de 2018. Em tal disciplina, tive acesso a alguns textos basilares a esse campo de estudos, entre os quais se encontra o artigo "Kinesiology's inconvenient truth: the physical cultural studies imperative", escrito por David L. Andrews, em 2008. Nele, o autor busca refletir a respeito do doxa científico que constitui a formação da cinesiologia, nas universidades, e a crise epistemológica decorrente da hegemonia das ciências biológicas em relação às ciências sociais e humanas, no interior da área.

Andrews (2008) denomina de "verdade inconveniente" a predominância de valorização - em termos de recursos financeiros e de reconhecimento geral - do conhecimento hegemônico na cinesiologia, a partir de critérios pautados no quantitativo, no preditivo e no modelo tradicional de ciência. Ele afirma que as duas áreas (biológica e social) precisam uma da outra para a formação do campo maior, que é o da cinesiologia. Nesse sentido, o autor entende que

sem os Estudos Culturais Físicos, eles [da área biológica] são biologia integrativa (seja lá o que for); com os Estudos Culturais Físicos somos capazes de cumprir a promessa da Cinesiologia. Isso pode ser uma verdade inconveniente para alguns, mas que, como campo, negligenciamos por nossa conta e risco (Andrews, 2008, p. 59, tradução nossa).

10 Disciplina ofertada pelo Programa de Pós-graduação Associado em Educação Física UEM/ UEL, ministrada pela profa. Dra. Larissa Michelle Lara, com carga horária de 30 horas/aula. 
Em janeiro de 2019, visitei a Universidade de Maryland, nos Estados Unidos, e entrevistei o professor Dr. David L. Andrews ${ }^{11}$. Na ocasião, a entrevista ${ }^{12}$ foi orientada por meio de um roteiro com sete questões, as quais visavam suscitar reflexões acerca da origem do PCS, de sua disseminação em diversos países e das possíveis contribuições desse campo aos estudos de esporte e mídia, haja vista ser esse último tema de minha área de investigação acadêmica no doutorado.

Perguntei a Andrews a respeito da "verdade inconveniente" - discussão desenvolvida em publicação basilar ao surgimento do PCS (Andrews, 2008) -, buscando compreender se tal constatação ainda fazia sentido. Em resposta, ele afirmou que o domínio das ciências biológicas em relação às ciências sociais e humanas, na cinesiologia, permanece inabalável.Logo, a "verdade inconveniente" continua a existir. Andrews (2008) avalia que os professores/pesquisadores do PCS - como projeto em andamento, na busca por ampliar a visão da cinesiologia para um campo epistêmico e ontologicamente abrangente e inclusivo - precisam persistir na luta por equidade em termos de estrutura de currículos, recursos financeiros e reconhecimento de gestores da universidade.

Andrews esclarece que a Universidade de Maryland foi a primeira a sistematizar o PCS, a partir do currículo que compõe a cinesiologia. Daí, adverte o professor, resulta o destaque do PCS na universidade, como um projeto inovador, em profícuo desenvolvimento e institucionalmente demarcado. Afinal, apesar de não terem muitos recursos financeiros, têm professores/as que integram o PCS e que são altamente engajados com publicações acadêmicas, projetos e ensino junto a disciplinas do currículo básico da graduação e da pós-graduação ${ }^{13}$. Todavia, Andrews (2008) ressalta que, apesar dos avanços, eles poderiam encontrar-se em melhores condições acadêmicas.

Ainda a respeito do progresso do PCS, nos últimos anos, Andrews (2008) relata que esse campo de estudos tem se propagado considerável e progressivamente, tanto de maneira epistemológica quanto de forma geográfica ${ }^{14}$. Entre os pilares dessa expansão está, segundo ele, o olhar crítico para a cultura física de modo geral, e não apenas para o esporte, o que amplia os focos de investigação. Entretanto, Andrews (2008) reforça que o PCS não se restringe à mera análise da cultura física como objeto empírico, pois, para além disso, é necessário que a investigação esteja comprometida com o entendimento de como o poder e as relações de poder

11 Atualmente, desenvolvo estágio doutoral junto à Universidade de Maryland, sob a supervisão do Prof. Dr. David Andrews e da Profa. Dra. Shannon Jette.

12 Esse estudo integra o projeto de pesquisa A teoria Physical Cultural Studies na Sociologia do Esporte e para além dela: aportes para as subáreas sociocultural e pedagógica da educação física brasileira (Proc. 8363/2017-UEM), realizado na Universidade Estadual de Maringá, sob coordenação da Profa. Dra. Larissa Michelle Lara.

13 Das disciplinas ofertadas na Universidade de Maryland, citamos algumas delas, quais sejam: Physical Cultural Studies: Research E Writing Seminar, Research Design:Physical Cultural Studies; Physical Cultural Studies: Culture/Hegemony/Articulation; History of Sport and Physical Culture; Theories of Physical Cultural Studies; Feminist Physical Cultural Studies, entre outras.

14 A ampliação geográfica de pesquisadores/as que discutem o PCS pode ser percebida na obra organizada por Silk, Andrews e Thorpe (2017), em que figuram pesquisadores dos seguintes países: Estados Unidos, Reino Unido, Austrália, Canadá, Nova Zelândia, Suécia, Japão, China, Itália e Brasil. 
operam dentro e por meio da cultura física analisada. Portanto, para ele, estudar a cultura física não significa estar, necessariamente, realizando um estudo na linha dos Estudos Culturais Físicos.

Na parte final da entrevista, os questionamentos realizados a Andrews (2008) voltaram-se aos estudos de esporte e mídia no PCS, sobretudo por identificar nessa abordagem aproximações com as pesquisas que tenho feito no decorrer de minha trajetória acadêmica. Tais aproximações revelam-se, especialmente, no ensejo de desvendar como as relações de poder operam na sociedade, por meio da articulação entre o esporte e a mídia (Greenberg et al., 2005; Silk, 2011; Andrews, 2012; Silk, Francombe-Webb e Andrews, 2014; Andrews, Lopes e Jackson, 2015; Hawzen e Newman, 2017).

Visando compreender a dinâmica de pesquisas sobre esporte e mídia no interior do PCS, perguntei a Andrews acerca de sua percepção em relação à contribuição do PCS para tal área. Em resposta, ele explica que estudos a respeito da representação e ideologia da mídia têm surgido com menos frequência, pois o foco das pesquisas do PCS, nos últimos anos, tem se dado em investigações de cunho etnográfico que envolvem o corpo, cujas experiências são amplamente valorizadas. Talvez isso ocorra, segundo ele, por motivos relacionados ao mercado de trabalho americano, pois, na disputa por vagas de empregos nas universidades, têm sido vantajosos os estudos experimentais etnográficos em relação aos estudos de mídia. Entretanto, Andrews (2008) reforça que o PCS é um projeto empiricamente complexo e não tem um único foco; o PCS constitui-se como sensibilidade que abarca, sobretudo, a cultura e as atividades físicas. Por essa razão, considera importante a configuração de uma amplitude temática. $\mathrm{O}$ pesquisador avalia que, para que um estudo sobre esporte e mídia esteja de acordo com os princípios do PCS, deve ter um objetivo político com a intenção de tornar transparente a política de representações populares.

Finalizando a entrevista, Andrews (2008) afirma que é fundamental o aumento no número de pesquisas nos Estudos Culturais Físicos que se voltem à decodificação dos discursos midiáticos, pelo público que os recebe, ainda que ele reconheça a dificuldade de executar uma pesquisa nesse sentido. Daí é possível depreender que a realização de pesquisas acerca do esporte e mídia, orientadas pelo PCS, pode resultar no desenvolvimento de temas ainda pouco explorados por esse complexo campo de investigação, contribuindo para agregar elementos à discussão da cultura física.

\section{REFLEXÕES ACERCA DOS ESTUDOS CULTURAIS FÍSICOS: CONTRIBUIÇÕES PARA O NOSSO CAMPO INVESTIGATIVO}

Os Estudos Culturais Físicos são um campo complexo e abrangente que tem nos instigado, tocado nossas subjetividades e nos levado a debruçar sobre seus referenciais teóricos, com vistas à compreensão da sensibilidade presente nas discussões que permeiam essa abordagem. Logo, o PCS tem influenciado nossas ações, nossas práticas pedagógicas e pesquisas acadêmicas. 
O PCS traz contribuições para pensarmos o ensino que perpassa a educação física, em especial aquele relacionado à educação em nível superior - no qual estamos inseridos/as como professores/as em universidades públicas brasileiras. A produção do PCS, em seu sentido pedagógico (Bush and Silk, 2010; Rich, 2011; Burrows e De Pian, 2017; Rich e Sandlin, 2017), convida-nos a (re)pensar a construção de currículos, a concepção de planos de ensino e a proposição de aulas pelo viés crítico e inovador, especificamente em meio às ideias de Paulo Freire e Henry Giroux, cujo foco recai em modos de levar "indivíduos e grupos a discernirem, a desafiarem e, potencialmente, a transformarem as estruturas e as relações de poder existentes, à medida que são manifestadas e experimentadas no complexo campo da cultura física" (Silk, Andrews e Thorpe, 2017, p. 8, tradução nossa).

Nesse sentido, com o PCS, impulsionamo-nos em direção a uma educação pública centrada na contestação de fronteiras acadêmicas tradicionais (Francombe, 2013; Francombe-Webb, Silk e Bush, 2017) e na projeção de espaços que construam subjetividades com potencial para pensar e agir, considerando-se as operações sociais de poder (Giroux,1997, 2013; Monteiro, 2017; Freire, 2018a, 2018b). Em complemento, essa maneira de ensinar está fundamentada em uma "pedagogia pública projetada para impactar as comunidades de aprendizado dentro da academia, nas salas de aula e em públicos mais amplos"(Silk, Andrews e Thorpe, 2017, p. 8, tradução nossa).

Outro aspecto importante diz respeito à dimensão autorreflexiva da pesquisa no PCS, constituída pelo compromisso subjetivo - moral e político - dos/as pesquisadores/as que o integram. Isso ocorre, pois, ao "fazer" Estudos Culturais Físicos (no sentido de colocá-los em ação), os/as intelectuais explicitam suas escolhas no interior da pesquisa, por meio de uma abordagem crítica com objetivos humanistas, evitando-se, assim, um suposto objetivismo isento de valores, disseminado pelas ciências positivistas (Silk, Andrews e Thorpe, 2017). Nessa perspectiva, o selfé, portanto, situado e incorporado nas relações postas na pesquisa, o que reflete, também, as decisões reflexivas junto ao desenvolvimento investigativo, à medida que são reconhecidas as necessidades de mudança em resposta às condições institucionais, históricas, sociais e/ou culturais enfrentadas no ato científico.

Para além desses aspectos supracitados, destacamos também, no PCS, a preocupação em olhar para os elementos da cultura física como locais em que as relações de poder operam e as injustiças e desigualdades sociais têm terreno fértil para aflorar e se propagar. Afinal, o PCS analisa como os corpos (in)ativos se tornam organizados, representados e experimentados em relação às operações de poder social, em todas as inumeráveis formas da cultura física - incluindo, mas não se restringindo às práticas relacionadas ao esporte, ao exercício, à saúde, à dança e ao movimento. Assim, o PCS identifica o papel desempenhado pela cultura física na reprodução de normas e diferenças impostas por hierarquias sociais (Andrews, 2008).

Nesse sentido, o PCS é um projeto político em que os/as pesquisadores/ as visam compreender os efeitos do poder e das relações de poder, uma vez que partem do pressuposto de que as sociedades se moldam por linhas de diferenciação hierárquicas, cujas diferenças se manifestam na existência de desigualdades ou injustiças culturais, vantagens ou desvantagens, habilitações ou restrições, 
empoderamentos ou desempoderamentos. $\mathrm{Na}$ medida em que, no interior da cultura física, o poder social se manifesta e opera em locais de luta política que são mutáveis, torna-se necessária a observação atenta desse dinamismo no sentido de melhor organização das estratégicas adotadas no PCS (Andrews, 2008; Silk e Andrews, 2011; Silk, Andrews e Thorpe, 2017). Por isso, os/as pesquisadores/as do PCS, comprometidos/as com a progressiva mudança social, buscam promover ações de intervenção junto à sociedade, em espaços cujas diferenças são latentes, no intuito de minimizar discrepâncias.

Por meio das narrativas autoetnográficas apresentadas neste artigo, com enfoques distintos em termos de experiências, é possível identificar que a sensibilidade autorreflexiva, pedagógica e política que permeia o PCS tem impulsionado nossas pesquisas e nossas práticas docentes. Dito de outro modo, afirmamos que esse campo de estudos tem contribuído para a reconfiguração de nosso embodiment, o que afeta a forma como ensinamos e pesquisamos na universidade. Assim, consideramos que a sensibilidade dos Estudos Culturais Físicos pode contribuir para uma prática de ensino, pesquisa e extensão abrangente, inclusiva e socialmente comprometida com a sociedade, no contexto brasileiro e, de modo específico, na educação física.

\section{CONSIDERAÇÕES FINAIS}

As experiências descritas nas narrativas autoetnográficas desenvolvidas neste estudo revelam, de certo modo, como os Estudos Culturais Físicos - campo investigativo, complexo e amplo - impactam diretamente nossas subjetividades e práticas acadêmicas. Ao longo das narrativas, discorremos acerca de nossa aproximação e identificação com o PCS, especialmente em seus aspectos pedagógicos e políticos, revelando como nosso embodiment tem sido ressignificado por esse campo, assim como tem impactado nossos modos de ensinar e fazer pesquisa, na universidade. Em complemento, o PCS direciona nosso olhar, ainda, para a educação física brasileira, levando-nos a refletir acerca do quão profícuo pode ser esse diálogo no sentido de uma educação física engajada, crítica e orientada em ações voltadas à democratização do acesso à cultura física.

Ao pensarmos na construção gradativa de um diálogo profícuo entre o PCS e a educação física no contexto brasileiro, lembramo-nos de parte da entrevista realizada com o professor David Andrews, quando o indagamos a respeito da possibilidade de existência dos Estudos Culturais Físicos latino-americanos - no sentido de pensarmos como essa abordagem se constituiria, a partir de características locais próprias. Em resposta, ele menciona que a existência do PCS latino-americano, definitivamente, seria viável e necessária, haja vista que a pesquisa no PCS teria, relativamente, mais fidedignidade se fosse desenvolvida por pesquisadores/ as que estivessem imersos/as no contexto local, mormente pelo fato de o PCS ser, fundamentalmente, contextual. Sobretudo, para ele, seria preciso criar uma rede de conexão entre as pessoas que já estão produzindo estudos na linha do PCS brasileiro ou do PCS latino-americano, a fim de verificar se existe coerência para se pensar em um projeto mais amplo. 
As narrativas autoetnográficas que aqui exploramos representam formas singulares de interlocução entre o PCS e a educação física no Brasil, as quais tendem, gradativamente, a ser adensadas por novas experiências e por outros interlocutores. O PCS nos impulsiona a lutar por uma educação física que vise contribuir, de maneira significativa e efetiva, para o desenvolvimento de pessoas e de comunidades por meio de práticas de intervenção social que respeitem os corpos diversos (gênero, deficiência, etnia, raça, habilidades, outros). Além disso, o PCS auxilia a nos mantermos vigilantes acerca das operações de poder que recaem sobre os corpos (in)ativos e que ocorrem nos meandros dos vários elementos constituintes da cultura física. Quiçá, poderemos, em um futuro próximo, ter elementos para potencializar o surgimento dos Estudos Culturais Físicos brasileiros ou com tons latino-americanos no contexto da educação física brasileira. A ideia não é meramente reproduzir esse campo de estudos na nossa realidade, mas sim estudá-lo, esmiuçá-lo, escavá-lo, no sentido de interlocuções que possam impactar nossa subjetividade, nossos modos de ensinar e pesquisar e nosso engajamento coletivo, com apropriações locais, culturais e identitárias, pensadas contextualmente em suas próprias relações de poder.

\section{REFERÊNCIAS}

ANDREWS,D. L. Kinesiology's inconvenient truth: the physical cultural studies imperative. Quest, v. 60, n. 1, p. 45-62, 2008. https://doi.org/10.1080/00336297.2008.10483568

ANDREWS, D. L. Reflections on communication and sport: on celebrity and race. Communication \& Sport, v. 1, n. 1-2, p. 151-163, 2012. https://doi. org/10.1177\%2F2167479512468869

ANDREWS, D. L.; LOPES, V. B.; JACKSON, S. J. Neymar: sport celebrity and performative cultural politics. In: MARSHALL, P. D.; REDMOND, S. A companion to celebrity. Londres: John Wiley \& Sons, 2015. p. 421-439.

ANDREWS,D.L.; SILK,M.L.Physical Cultural Studies on Sport.In: GIULIANOTTI, R. (org.). Routledge Handbook of the Sociology of Sport. Londres: Routledge, 2015. p. 83-93. BRASIL. Lei no 9.394, de 20 de dezembro de 1996. Estabelece as diretrizes e bases da educação nacional. Diário Oficial da União, Brasília, 23 dez. 1996.

BRASIL. Lei no 10.639, de 9 de janeiro de 2003. Altera a Lei no 9.394, de 20 de dezembro de 1996, que estabelece as diretrizes e bases da educação nacional, para incluir no currículo oficial da Rede de Ensino a obrigatoriedade da temática "História e Cultura Afro-Brasileira”, e dá outras providências. Diário Oficial da União, Brasília, 10 jan. 2003. BRASIL. Conselho Nacional de Educação. Conselho Pleno. Parecer no 3, de 10 de março de 2004. Estabelece Diretrizes Curriculares Nacionais para a Educação das Relações Étnico-Raciais e para o Ensino de História e Cultura Afro-Brasileira e Africana. Diário Oficial da União, Brasília, 19 maio 2004.

BRASIL. Lei no 11.645 , de 10 de março de 2008. Inclui no currículo oficial da rede de ensino a obrigatoriedade da temática "História e Cultura Afro-brasileira e Indígena". Diário Oficial da União, Brasília, 11 mar. 2008. 
BURROWS, L.; DE PIAN, L. Physical education, policy and embodied pedagogies. In: SILK, M. L.; ANDREWS, D. L.; THORPE, H. (org.). Routledge handbook of Physical Cultural Studies. Londres: Routledge International Handbooks, 2017. p. 423-431.

BUSH, A. J.; SILK, M. L. Towards an evolving critical consciousness in coaching research: the Physical Pedagogic Bricolage. International Journal of Sports Science \& Coaching, v. 5, n. 4, p. 551-565, 2010. https://doi.org/10.1260\%2F1747-9541.5.4.551 CALHA, A. O uso da autobiografia na educação de adultos: modos narrativos de valorização e legitimação da experiência profissional. Revista Brasileira de Educação, v. 22, n. 71, e227168, 2017. https://doi.org/10.1590/s1413-24782017227168. http:// dx.doi.org/10.20952/revtee.v12i28.10161

FRANCOMBE, J. Methods that move: a physical performative pedagogy of subjectivity. Sociology of Sport, v. 30, n. 3, p. 256-273, 2013. https://doi.org/10.1123/ssj.30.3.256 FRANCOMBE-WEBB, J.; SILK, M. L.; BUSH, A. Critical corporeal curricula, praxis and change. In: SILK, M. L.; ANDREWS, D. L.; THORPE, H. (org.). Routledge handbook of physical cultural studies. Londres: Routledge International Handbooks, 2017. p. 558-567.

FREIRE, P. Pedagogia da autonomia: saberes necessários à prática educativa. 56. ed. Rio de Janeiro/São Paulo: Paz e Terra, 2018a.

FREIRE, P. Pedagogia do oprimido. 65. ed. Rio de Janeiro/São Paulo: Paz e Terra, 2018b.

FULLAGAR, S. P. A physical cultural studies perspective on physical (in)activity and health inequalities: the biopolitics of body practices and embodied movement. Tempos e Espaços em Educação, v. 12, n. 28, p. 63-76,jan./mar. 2019. https://doi.org/10.20952/ revtee.v12i28.10161

GIARDINA, M.D.; NEWMAN,J.I. Physical cultural studies and embodied research acts. Cultural Studies: Critical Methodologies, v. 11, n. 6, p. 523-534, 16 nov. 2011a. https://doi.org/10.1177\%2F1532708611426107

GIARDINA, M.; NEWMAN,J.I.What is this "physical" in Physical Cultural Studies? Sociology of Sport Journal, v. 28, n. 1, p. 36-63, 2011b. https://doi.org/10.1123/ ssj.28.1.36

GIROUX, H. A. Os professores como intelectuais: rumo a uma pedagogia crítica da aprendizagem. Porto Alegre: Artes Médicas, 1997.

GIROUX, H. A.Praticando Estudos Culturais nas faculdades de educação. In: SILVA, T. T. (org.). Alienígenas na sala de aula: uma introdução aos estudos culturais em educação. 11. ed. Petrópolis: Vozes, 2013. p. 83-100.

GREENBERG,J.et al. Negotiated order and strategic inaction in television coverage of the olympics. In: PAWLUCH, D.; SHAFFIR, W.; MIALL, C. Doing ethnography: studying everyday life. Toronto: Canadian Scholars' Press, 2005. p. 288-299.

HALL, S. A centralidade da cultura: notas sobre as revoluções de nosso tempo. Educação e Realidade, v. 22, n. 2, p. 15-46, jul.-dez. 1997. 
HALL, S. Quem precisa de identidade? In: SILVA, T. T. (org.). Identidade e diferença: a perspectiva dos estudos culturais. Petrópolis: Vozes, 2000. p. 103-133.

HALL, S. Da diáspora: identidade e mediações culturais. Belo Horizonte: UFMG; Brasília: Representação da UNESCO no Brasil, 2003.

HAWZEN, M. G.; NEWMAN, J. I. The gospel according to Tim Tebow: sporting celebrity, whiteness, and the cultural politics of Christian fundamentalism in America. Sociology of Sport Journal, v. 34, n. 1, p. 12-24, 2017. https://doi.org/10.1123/ ssj.2016-0079

INGHAM, A. G. Toward a Department of Physical Cultural Studies and an end to tribal warfare. In: FERNANDEZ-BALBOA, J.-M. (org.). Critical Postmodernism in Human Movement, Physical Education, and Sport. Suny Series on Sport, Culture, and Social Relations. Albany: State University of New York Press, 1997. p. 157-182.

KING-WHITE, R. Oh Henry!: Physical Cultural Studies" Critical Pedagogical Imperative. Sociology of Sport Journal, v. 29, n. 3, p. 385-408, 2012. https://doi. org/10.1123/ssj.29.3.385

LARA, L. M. Pesquisa e ensino em corpo, cultura e ludicidade em tempos de adversidades. In: LARA, L. M.; LOPES, B. R.; SOUZA, V. F. M. (org.). SIMPÓSIO ESTUDOS CULTURAIS NA EDUCAÇÃO FÍSICA: 15 ANOS DE PESQUISA EM CORPO, CULTURA E LUDICIDADE, 1., 2019, Maringá. Anais [...]. Maringá: GPCCL, 2019. p. 59-66.

LARA, L. M. et al. Resenha de Routledge Handbook of Physical Cultural Studies. Revista Brasileira de Ciências do Esporte, v. 41, n. 2, p.229-230, abr./jun.2019. https:// doi.org/10.1016/j.rbce.2018.05.002

LARA, L. M.; RICH, E. Os estudos de cultura física na Universidade de Bath-Reino Unido: dimensões de uma abordagem muito além da fisicalidade. Movimento, v. 23, n. 4, p. 1311-1324, out./dez. 2017. https://doi.org/10.22456/1982-8918.74326

MARANI, V.H.O (re)conhecimento do corpo nos estudos culturais físicos: a pesquisa (in)corporada como meio para a visibilidade social. In: LARA, L. M.; LOPES, B. R.; SOUZA, V.F.M. (org.). SIMPÓSIO ESTUDOS CULTURAIS NA EDUCAÇÃO FÍSICA: 15 ANOS DE PESQUISA EM CORPO, CULTURA E LUDICIDADE, 1., 2019, Maringá. Anais [...]. Maringá: GPCCL, 2019. p. 35-42.

MARKULA, P.; DENISON, J. Sport and the Personal Narrative. In: ANDREWS, D. L.; MASON, D. S.; SILK, M. L. (org.). Qualitative methods in sports studies. Oxford e Nova York: Berg, 2005. p. 165-184.

MARKULA, P.; SILK, M. Qualitative research for physical culture. Londres: Palgrave MacMillan, 2011.

MARQUES, J. P. Experiências do corpo nos estudos culturais e saberes formativos na educação física: subjetivação, institucionalização, saúde e disciplina. In: LARA, L. M.; LOPES, B. R.; SOUZA, V. F. M. (org.). SIMPÓSIO ESTUDOS CULTURAIS NA EDUCAÇÃO FÍSICA: 15 ANOS DE PESQUISA EM CORPO, CULTURA E LUDICIDADE, 1., 2019, Maringá. Anais [...]. Maringá: GPCCL, 2019. p. 125-133. 
MONTEIRO, M. V. P. A construção identitária nas aulas de educação física. Revista Brasileira de Educação, v. 22, n. 69, p. 339-359, abr.-jun. 2017. https://doi.org/10.1590/ s1413-24782017226918

REGO, T. C. Trajetória intelectual de pesquisadores da educação a fecundidade do estudo dos memoriais acadêmicos. Revista Brasileira de Educação, v. 19, n. 58, p. 779800, jul.-set. 2014. https://doi.org/10.1590/S1413-24782014000800013

$\mathrm{RICH}$, E. Exploring the relationship between pedagogy and Physical Cultural Studies: the case of new health imperatives in schools. Sociology of Sport Journal, v. 28, n. 1, p. 64-84, 2011. https://doi.org/10.1123/ssj.28.1.64

RICH, E.; SANDLIN,J. A.Physical cultural studies and public pedagogies. In: SILK, M. L.; ANDREWS, D. L.; THORPE, H. (org.). Routledge handbook of Physical Cultural Studies. Londres: Routledge International Handbooks, 2017. p. 549-557.

SÁ,A. B. S.Physical Cultural Studies: reflexões acerca da produção de conhecimento em mídia esportiva. In: LARA, L. M.; LOPES, B. R.; SOUZA, V. F. M.(org.). SIMPÓSIO ESTUDOS CULTURAIS NA EDUCAÇÃO FÍSICA: 15 ANOS DE PESQUISA EM CORPO, CULTURA E LUDICIDADE, 1., 2019, Maringá. Anais [...]. Maringá: GPCCL, 2019. p. 134-140.

SILK, M. L. Towards a Sociological Analysis of London 2012. Sociology, v. 45, n. 5 , p. 733-748, 2011. https://doi.org/10.1177\%2F0038038511413422

SILK, M. L.; ANDREWS, D. L. Toward a Physical Cultural Studies. Sociology of Sport Journal, v. 28, n. 1, p. 4-35, 2011. https://doi.org/10.1123/ssj.28.1.4

SILK, M. L.; ANDREWS, D. L.; THORPE, H. (org.). Routledge handbook of Physical Cultural Studies. Londres: Routledge International Handbooks, 2017.

SILK, M. L.; BUSH, A.; ANDREWS, D. L. Contingent intellectual amateurism, or, the problem with evidence-based research. Journal of Sport and Social Issues, v. 34, n. 1, p. 105-128, 2010. https://doi.org/10.1177\%2F0193723509360112

SILK, M. L.; FRANCOMBE-WEBB, J.; ANDREWS, D. L. The corporate constitution of national culture: the mythopoeia of 1966 . Continuum, v. 28, n. 5, p. 720-736, 2014. https://doi.org/10.1080/10304312.2014.941326

SILVA, P. P.; OLIVEIRA, A. M. P.; SOUZA, E. C. "Mais parece um saca-rolha que um caminho!": identidades contingentes de pesquisadora em ciências biológicas a pesquisadora em ciências humanas. Revista Brasileira de Educação, v. 23, e230092, 2018. https://doi.org/10.1590/s1413-24782018230092

SMITH, B. Narrative inquiry and autoethnography. In: SILK, M. L.; ANDREWS, D. L.; THORPE, H. (org.). Routledge handbook of physical cultural studies. Londres: Routledge International Handbooks, 2017. p. 505-514.

THORPE, H.; BARBOUR, K.; BRUCE, T. "Wandering and Wondering”: theory and representation in feminist physical cultural studies. Sociology of Sport Journal, v. 28, n. 1, p. 106-134, mar. 2011. https://doi.org/10.1123/ssj.28.1.106

THORPE, H.; MARFELL, A. Feminism and the Physical Cultural Studies Assemblage: revisiting debates and imagining new directions. Leisure Sciences, v. 41, n. 1-2, p. 17-35, 23 jan. 2019. https://doi.org/10.1080/01490400.2018.1539684 


\section{SOBRE OS AUTORES}

Ariane Boaventura da Silva Sá é doutoranda em educação física pela Universidade Estadual de Maringá (UEM).

E-mail: ariane.boaventura@hotmail.com

Vitor Hugo Marani é doutor em educação física pela Universidade Estadual de Maringá (UEM). Professor da Universidade Federal de Mato Grosso (UFMT).

E-mail: vitormarani@ufmt.br

Larissa Michelle Lara é doutora em educação pela Universidade Estadual de Campinas (UNICAMP). Professora da Universidade Estadual de Maringá (UEM).

E-mail:mlara@uem.br

Conflitos de interesse: Os autores declaram que não possuem nenhum interesse comercial ou associativo que represente conflito de interesses em relação ao manuscrito.

Financiamento: Coordenação de Aperfeiçoamento de Pessoal de Nível Superior - Brasil (CAPES) - Código de Financiamento 001, como desdobramento do estágio pós-doutoral sênior da terceira autora na Universidade de Bath (Reino Unido) e da participação no Programa de Doutorado Sanduíche no Exterior (PDSE) do segundo autor na Universidade de Maryland (Estados Unidos).

Contribuições dos autores: Administração do Projeto: Sá, A.B.S.; Marani, V.H.; Lara, L.M. Análise Formal: Sá, A.B.S.; Marani, V.H.; Lara, L.M. Conceituação: Sá, A.B.S.; Marani, V.H.; Lara, L.M. Curadoria de Dados: Sá, A.B.S.; Marani, V.H.; Lara, L.M. Escrita - Primeira Redação: Sá, A.B.S.; Marani, V.H.; Lara, L.M. Escrita - Revisão e Edição: Sá, A.B.S.; Marani, V.H.; Lara, L.M. Investigação: Sá,A.B.S.; Marani, V.H.; Lara, L.M. Metodologia: Sá, A.B.S.; Marani, V.H.; Lara, L.M. Obtenção de Financiamento: Marani, V.H.; Lara, L.M. Recursos: Sá, A.B.S.; Marani, V.H.; Lara, L.M. Software: Sá, A.B.S.; Marani, V.H.; Lara, L.M. Supervisão: Lara, L.M. Validação e Visualização: Sá, A.B.S.; Marani, V.H.; Lara, L.M. 